\title{
Macrophage migration inhibitory factor enhances autophagy by regulating ROCK1 activity and contributes to the escape of dendritic cell surveillance in glioblastoma
}

\author{
SHUGANG XU ${ }^{1,2^{*}}, \mathrm{XING} \mathrm{GUO}^{1 *}, \mathrm{XIAO} \mathrm{GAO}^{1}, \mathrm{HAO}^{\mathrm{X}} \mathrm{XE}^{1}$, JINSEN ZHANG ${ }^{1}$, \\ XIAOFAN GUO $^{1}$, WEI QIU ${ }^{1}$, PING ZHANG ${ }^{1}$ and GANG LI ${ }^{1,3}$ \\ ${ }^{1}$ Department of Neurosurgery, Qilu Hospital of Shandong University, Jinan, Shandong 250012; \\ ${ }^{2}$ Department of Neurosurgery, Dezhou People's Hospital, Dezhou, Shandong 253014; \\ ${ }^{3}$ Brain Science Research Institute, Shandong University, Jinan, Shandong 250012, P.R. China
}

Received July 3, 2016; Accepted September 8, 2016

DOI: 10.3892/ijo.2016.3704

\begin{abstract}
Macrophage migration inhibitory factor (MIF) is highly expressed in glioblastoma, promoting malignant progression and suppresses immune surveillance. However, the mechanism underlying its biological roles in human glioblastoma and the capability of MIF to escape dendritic cell (DC) surveillance remain poorly understood. In the present study, we found that recombinant human MIF (rhMIF) activated the RhoA-ROCK1 pathway and simultaneously upregulated F-actin fibre formation. Additionally, we showed that rhMIF increased autophagy in glioblastoma cells, and knockdown of endogenic MIF suppressed autophagy. In glioma specimens, MIF expression was significantly correlated with LC3B levels. Moreover, we confirmed that the activity of Rho-associated coiled-coil containing kinase (ROCK)1 played a crucial role in MIF-induced autophagy. Y26736, a ROCK1 inhibitor, blocked the MIF-mediated increase in migration and colony formation in glioblastoma multiforme (GBM) cells. Furthermore, exogenous rhMIF suppressed the migration of both immature DCs (iDCs) and mature DCs (mDCs). Addition of rhMIF during the maturation process of iDCs impaired the expression of co-stimulatory markers. Taken together, our results identified ROCK1 as a critical mediator of MIF-induced autophagy and the immunosuppressive effect of MIF on DC surveillance in glioblastoma.
\end{abstract}

Correspondence to: Dr Gang Li, Department of Neurosurgery, Qilu Hospital of Shandong University, 107 Wenhua Western Road, Jinan, Shandong 250012, P.R. China

E-mail: ligangqiluhospital@163.com

*Contributed equally

Key words: macrophage migration inhibitory factor, autophagy, Rho-associated coiled-coil containing kinase 1, dendritic cell, glioblastoma

\section{Introduction}

Glioblastoma multiforme (GBM) is the most prevalent and aggressive malignant tumour of the central nervous system. Despite progress in therapeutic strategies combining surgery, radio-chemotherapy and immunotherapy, patients with GBM still have a dismal prognosis $(1,2)$. Therefore, elucidation of the molecular mechanisms underlying the development and progression of GBM is needed to explore new specific therapeutic strategies.

Macrophage migration inhibitory factor (MIF) was first identified due to its role in inhibition of macrophage migration (3). After decades of research, MIF has been shown to play a vital role in both immune response and tumourigenesis (4). In addition to inhibition of macrophage migration, MIF blocks NK cell activity (5), decreases acute inflammation and mediates chronic inflammation, which partly promotes tumourigenesis (6-8). As a promotor of tumourigenesis, MIF is strongly expressed in various types of malignant cancers, including GBM $(9,10)$. MIF exhibits a broad spectrum of pro-neoplastic activities, enhancing tumour cell proliferation (11), inducing angiogenesis (12), and reducing tumour cell death by inhibition of p53 (13). In recent studies, MIF has been shown to contribute to malignant progression of GBM. These results indicate that MIF is a promising target for anti-GBM therapy. However, the signalling pathways activated by MIF have not been completely elucidated thus far.

Autophagy is a highly conserved cellular catabolic pathway that monitors, degrades and recycles intracellular proteins and organelles in lysosomes to support metabolism and promotes cell survival $(14,15)$. It is believed to play a contextual role in cancer (16). In $40-75 \%$ of specific cancers, such as human prostate, breast, and ovarian cancers, the essential autophagy gene ATG6/BECN1 was lost (17-19). However, in many cancers, autophagy was a tumour promoter that was involved in tumour initiation and development (20). High levels of autophagy in glioma, especially under stressful microenvironments, promote malignant progression. To date, autophagy has been suggested to be a potential target for glioma therapy. 
Rho-associated coiled-coil containing kinases (ROCKs) are serine/threonine kinases that are central regulators of the actomyosin cytoskeleton (20). There are two mammalian ROCK homologs, ROCK1 and 2. ROCKs were initially recognized as activated Rho GTPase-binding proteins. The Rho GTPase family is known for its regulation of actin cytoskeleton organization and dynamics (21). RhoA and B, which belong to the Rho GTPase proteins, are the best characterized ROCK regulators $(22,23)$. Activation of ROCKs promotes the formation of stress fibres and actomyosin contraction via phosphorylation of numerous downstream target proteins, including the myosin regulatory light chain (MLC) and the myosin-binding subunit (MYPT1) (24-26). In addition, extensive studies have demonstrated that ROCK1 has a diverse range of functions in tumourigenesis, including cell contraction, migration, apoptosis, survival, and proliferation.

Dendritic cells (DCs) are professional antigen-presenting cells of the immune system that are derived from hematopoietic progenitor cells (HPCs) in the bone marrow. They initiate and modulate the immune response (27). In the periphery, immature DCs (iDCs) derived from proliferating progenitors capture and process antigens. As a consequence of antigen deposition and inflammation, DCs begin to mature, expressing lymphocyte co-stimulatory molecules, migrating to lymphoid organs and secreting cytokines to initiate immune responses. Many studies have shown that DCs can identify and attack infiltrating tumour cells to control tumour regrowth through immunological memory and immune surveillance $(28,29)$. These studies and others have challenged the traditional notion of CNS 'immune privilege', which is an imprecise characterization of the CNS immune environment (30). Immunotherapy for GBM appears to be a meaningful treatment approach to promote long-term survival, and DCs play a vital role in this strategy (31).

In the present study, we identified MIF as a promoter of autophagy in GBM cells. Furthermore, we verified that ROCK1 was involved in MIF-induced progression of glioma. In addition, we explored the potential immune functions of MIF and found that MIF played an inhibitory role in the immune response of DCs. Our research highlighted the different roles of MIF in glioma progression and the immune system response.

\section{Materials and methods}

Ethics statement. This study was approved by the Institutional Review Board of Shandong University and written informed consent was obtained from all patients, and the Hospital Ethics Committee approved the experiments.

Tissue samples and cell lines. Human glioma cell lines (U87 and U251) were purchased from the Cell Bank of the Chinese Academy of Sciences (Shanghai, China). Both U87 and U251 cells had been recently authenticated based on cross-species checks, DNA authentication and quarantine. The cell lines were grown in Dulbecco's modified Eagle's medium (DMEM; HyClone, Logan, UT, USA) supplemented with $10 \%$ fetal bovine serum (Gibco BRL, Gaithersburg, MD, USA) in a humidified incubator with $5 \% \mathrm{CO}_{2}$ at $37^{\circ} \mathrm{C}$. Six normal brain tissues were collected from patients undergoing internal decompression surgery following severe traumatic brain injury. Twenty-five human glioma tissues, including 12 low-grade glioma tissues (5 grade-I and 7 grade-II tumours) and 13 high-grade glioma tissues (3 grade-III and 10 grade-IV tumours) were obtained from the Department of Neurosurgery, Qilu Hospital of Shandong University.

Chemical reagents, siRNA and transfections. Recombinant human MIF (rhMIF; Peprotech, Inc., Rocky Hill, NJ, USA), Y27632 (Selleck Chemicals, Houston, TX, USA), IL-4, GM-CSF (both from Peprotech, Inc.), LPS (Sigma-Aldrich), CCL21 (Peprotech, Inc.) and DAPI (Beyotime Institute of Biotechnology, Shanghai, China) were obtained from indicated companies. siRNA negative control and siMIF were designed and purchased from GenePharma (Shanghai, China). The siRNA sequences used were the following: siRNA negative control forward, 5'-UUCUCCGAACGUGUCACGUTT-3' and reverse, 5'-ACGUGACACGUUCGGAGAATT-3'; siMIF forward, 5'-CCGAUGUUCAUCGUAAACATT-3' and reverse, 5'-UGUUUACGAUGAACAUCGGTT-3'. Cell transfection experiments were performed with nucleic acids using Lipofectamine 2000 (Invitrogen Life Technologies, Carlsbad, CA, USA) according to the manufacturer's instructions.

RhoA GTPase activity assay. The activity of RhoA and Rac1 GTPases in cell lysates from control and MIF-treated glioblastoma cells was assessed using a G-LISA ${ }^{\circledR}$ assay to measure the GTP-bound form of RhoA and Racl (Cytoskeleton, Inc., Denver, CO, USA). Briefly, glioblastoma cells were incubated with MIF (100 ng/ml, $30 \mathrm{~min})$. Cell lysates were incubated on RhoA and Racl GTPase affinity plates and color developed using HRP detection reagent mixture. Samples were read on a microplate reader (Bio-Rad, Berkeley, CA, USA).

$R N A$ isolation, reverse transcription, and quantitative real-time PCR. qPCR was conducted to measure the expression levels of DCs. Total RNA was isolated using RNAiso Plus (Takara). Total RNA $(0.5-1 \mu \mathrm{g})$ was reverse-transcribed with a ReverTra Ace qPCR RT kit (FSQ-101, Toyobo) according to the manufacturer's protocol to synthesize cDNA. Real-time PCR was performed using a SYBR Premix Ex Taq ${ }^{\mathrm{TM}}$ kit (Toyobo) with specific primers. The primers used were the following: CD80 forward, 5'-AAACTCGCATCTACTGGCAAA-3' and reverse, 5'-GGTTCTTGTACTCGGGCCATA-3'; CD83 forward, 5'-AAGGGGCAAAATGGTTCTTTCG-3' and reverse, 5'-GCA CCTGTATGTCCCCGAG-3'; CD86 forward, 5'-CTGCTCAT CTATACACGGTTACC-3' and reverse, 5'-GGAAACGTCGT ACAGTTCTGTG-3'. The reactions were performed by using a LightCycler 2.0 Instrument (Roche Applied Science, Mannheim, Germany). mRNA levels were normalized to GAPDH. All data for each sample were collected in triplicate. The fold changes were calculated by relative quantification $\left(2^{-\Delta \Delta \mathrm{Ct}}\right)$.

GFP-LC3 stable cell lines and quantitative GFP-LC3 analysis. To obtain U251 and U87 GFP-LC3 stable cell lines, we cloned LC3 inserts to the plenty-N-GFP vectors and generated lentiviruses by GenePharma. U87 and U251 cells were infected with the lentiviruses and selected stable clones with G418 (Sangon Biotech Co., Ltd., Shanghai, China). U87 and U251 GFP-LC3 stable cell lines were transfected with siMIF or treated with 
rhMIF and fixed in 4\% paraformaldehyde. GFP-LC3 puncta formation assay was determined by capturing images using Olympus microscope (DP72; Olympus, Tokyo, Japan). Cells with $\geq 5$ puncta were considered as GFP-LC3 puncta-positive cells. The percentage of GFP-LC3 puncta-positive cells was quantified by counting 200 GFP-LC3 stable cells.

Staining of F-actin fibre formation using rhodamine-phalloidin. Glioblastoma cells and DCs plated on 24-well plate were grown for $24 \mathrm{~h}$ and then cultured in serum-free medium for $16 \mathrm{~h}$. After incubating with or without rhMIF (100 ng/ml, $24 \mathrm{~h}$ ), glioblastoma cells were washed once with phosphate-buffered saline (PBS) and DCs were dropped on a glass slide. Then they were fixed with $4 \%$ formaldehyde in PBS for $10 \mathrm{~min}$. The fixed cells were washed twice with PBS and permeabilized with $0.3 \%$ Triton X-100 in PBS. Cells were then stained with rhodamine-phalloidin (Cytoskeleton, Inc.) in PBS and cell nuclei were counterstained with DAPI. Images were captured by Olympus microscope.

Western blot analysis. Total protein was extracted from tissues and cells using RIPA buffer (Beyotime Institute of Biotechnology) with $1 \%$ phenylmethyl sulfonyl fluoride, and protein concentration was determined by the BCA method (Beyotime Institute of Biotechnology). Proteins were separated using 10-15\% SDS-PAGE and transferred onto polyvinylidene difluoride membranes (Millipore, USA). The membranes were blocked by $5 \%$ skim milk blocking buffer for $1 \mathrm{~h}$ and then incubated in the primary antibodies at $4^{\circ} \mathrm{C}$ overnight. After washing with TBST, the blots were incubated with horseradish peroxidase-conjugated secondary antibodies at room temperature for $1 \mathrm{~h}$. Finally, protein bands were visualized by enhanced chemiluminescence (ECL) (Millipore) and detected using an ECL detection system (Thermo Fisher Scientific, Inc., Beijing, China) and quantified with Quantity One software. The following primary antibodies were used: rabbit anti-LC3B, p62 and p-MYPT1 were purchased from Cell Signaling Technology, Inc. (Danvers, MA, USA); rabbit anti-ROCK1 was purchased from Abcam (Cambridge, UK); rabbit anti-MYPT1 was purchased from ProteinTech Group, Inc. (Wuhan, China); rabbit anti-GAPDH was purchased from Goodhere Biotechnology Co., Ltd. (Hangzhou, China). The relative integrated density values were measured based on the GADPH protein as the control.

Immunohistochemistry. Paraffin-embedded samples were sliced and mounted on microscopic slides. Rabbit anti-MIF (1:200 dilutions), anti-LC3B (1:200 dilutions) and anti-CD1a (ZSGB-Bio, Beijing, China) antibodies were used as the primary antibodies. Heat-induced epitope retrieval was performed with a microwave in $10 \mathrm{mmol} / \mathrm{l}$ citric acid buffer at $\mathrm{pH}$ 7.2. The samples were incubated with the antibody overnight in a humidified chamber at $4^{\circ} \mathrm{C}$ followed by incubation with a horseradish peroxidase-conjugated secondary antibody (ZSGB-Bio). Finally, 3,3'-diaminobenzidine tetrahydrochloride (DAB) was used to reveal the signal. The total immunostaining score was estimated using both the percentage of positively stained tumour cells and the staining intensity. The percentage positivity was scored as ' 0 ' $(<5 \%$, negative), ' 1 ' (5-25\%, sporadic), ' 2 ' (25-50\%, focal), or ' 3 '
( $>50 \%$, diffuse). The staining intensity was scored as '0' (no staining), '1' (weakly stained), '2' (moderately stained), or ' 3 ' (strongly stained). Both the percentage of positive cells and the staining intensity were evaluated under double-blind conditions. The immunostaining score was calculated as the percentage positive score multiplied by the staining intensity score and ranged from 0 to 9 .

In vitro migration assay. DC migration was assessed as previously described (32). Briefly, the lower chamber of 24 Transwell plates with polycarbonate membranes and 5- $\mu \mathrm{m}$ pore size (Corning, Inc.) was filled with $200 \mu \mathrm{l}$ of RPMI-1640 media containing $10 \% \mathrm{FBS}$ and CCL21 (100 ng/ml). Next, iDC and mature $\mathrm{DC}(\mathrm{mDC})\left(1 \times 10^{5} / 100 \mu \mathrm{l}\right.$ medium) were seeded in the upper chamber, and plates were incubated for $2 \mathrm{~h}$ at $37^{\circ} \mathrm{C}$. Cells in the lower chamber were counted. Glioblastoma cell migration was evaluated using a Transwell chamber $(8-\mu \mathrm{m}$ pore size; Corning, Inc.). Cells that did migrate to the lower surface were fixed and stained with eosin solution and counted under a microscope (Olympus). Five random views were used to count the cells, and the independent experiments were repeated three times.

Colony formation assay. Glioblastoma cells (500) were seeded in 6-well plates and cultured for 4 weeks at DMEM containing $10 \%$ FBS with indicated treatments. Then the colonies were washed three times with PBS, and fixed with $75 \%$ ethanol for $10 \mathrm{~min}$, dried and stained with $0.1 \%$ crystal violet solution (Beyotime Institute of Biotechnology) for $10 \mathrm{~min}$. Images were taken and the colonies were counted under the light microscope.

Generation and culture of monocyte-derived DCs. PBMCs were isolated from leukocyte-enriched buffy coats of healthy volunteers by centrifugation with Ficoll-Paque Plus (Sigma-Aldrich). Monocytes were enriched from PBMCs by positive selection using anti-CD14-conjugated magnetic MicroBeads (Miltenyi Biotec). Monocytes were cultured at $1 \times 10^{6} / \mathrm{ml}$ in complete RPMI medium containing $1,000 \mathrm{U} / \mathrm{ml}$ GM-CSF and $500 \mathrm{U} / \mathrm{ml} \mathrm{IL-4}$ under $37^{\circ} \mathrm{C}, 5 \%$ humidified $\mathrm{CO}_{2}$ for 5 days to generate iDCs. In the middle of the 5 days, half of the medium was replaced by fresh medium containing cytokines. To induce maturation, $1 \mu \mathrm{g} / \mathrm{ml}$ LPS was added in iDCs for another 2 days. To investigate the influence of MIF on DC maturation, $100 \mathrm{ng} / \mathrm{ml} \mathrm{rhMIF}$ were added during or after the maturation.

Statistical analyses. Data analyses were conducted with SPSS 16.0 (SPSS, Inc., Chicago, IL, USA) and GraphPad Prism 5 (GraphPad Software, Inc., La Jolla, CA, USA). Data were analyzed using one-way ANOVA, Student's two-tailed $\mathrm{t}$-test. Data are presented as the mean \pm standard deviation (SD) of three independent experiments, followed by Dunnett's test for multiple comparisons of the means. All tests were two-tailed, and $\mathrm{p}<0.05$ was considered statistically significant.

\section{Results}

MIF activates the RhoA-ROCK1 pathway in glioblastoma cells. We first investigated whether RhoA-ROCK1 activity 

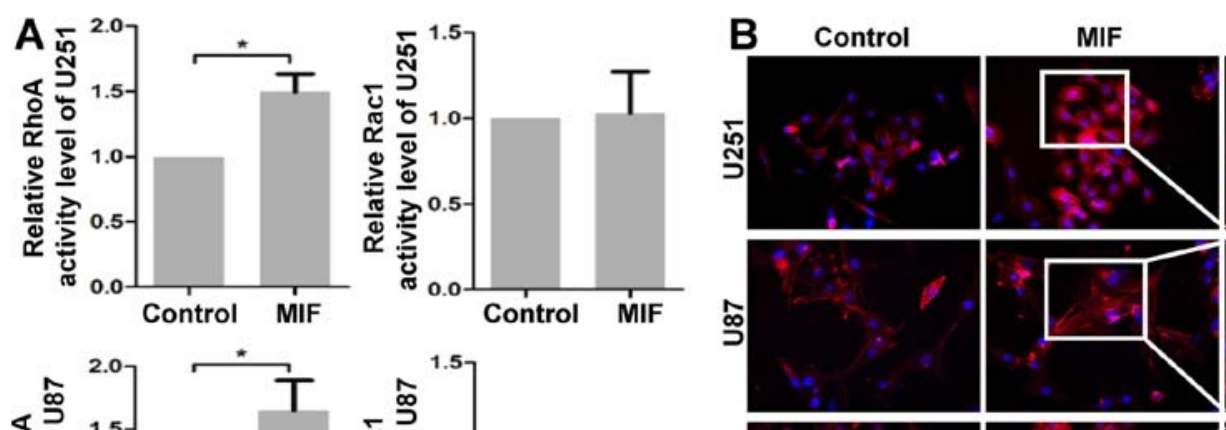

Magnification
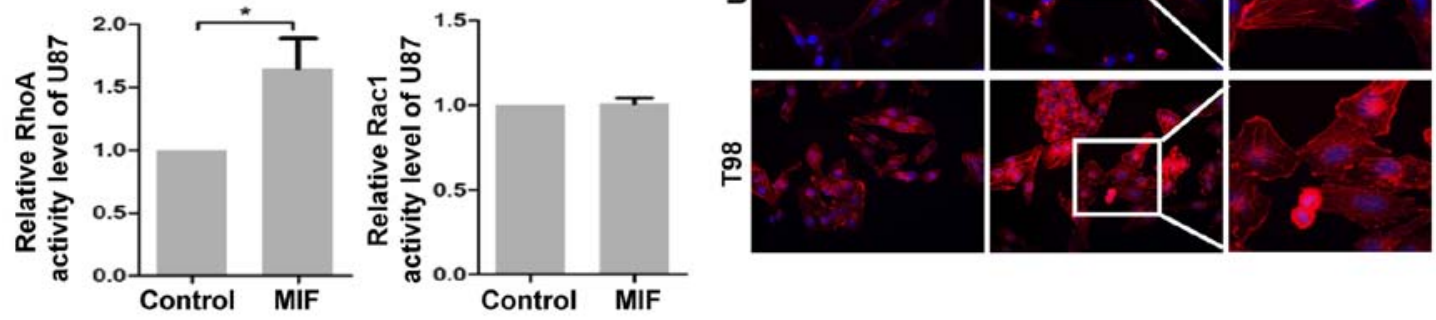

C
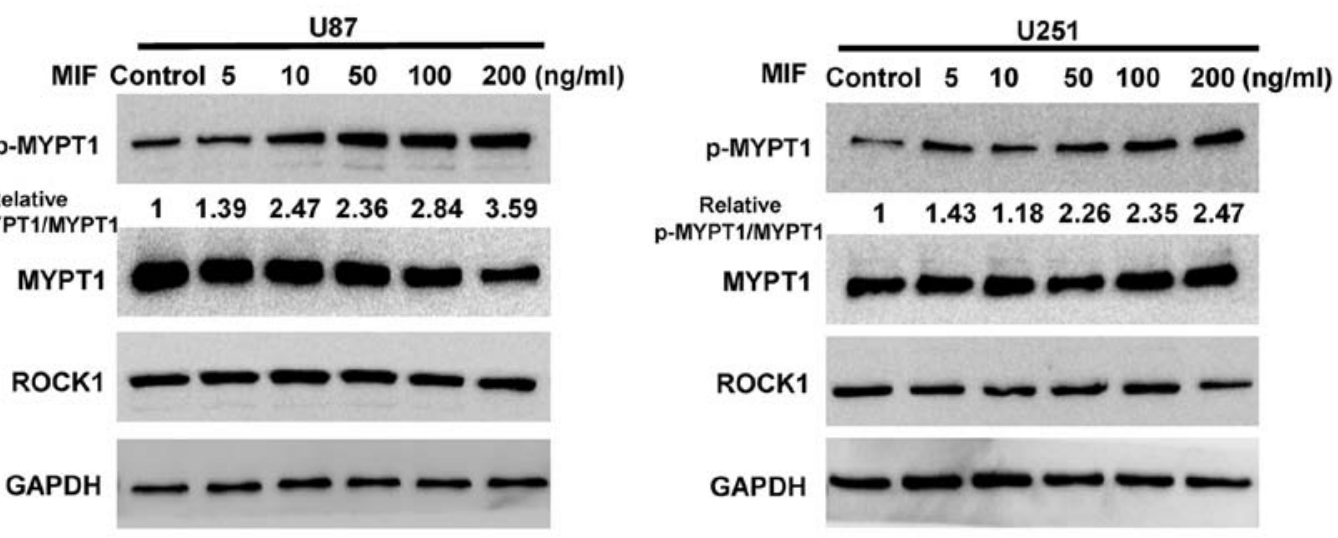

Figure 1. MIF promotes the activity of ROCK1. (A) U87 and U251 cells were untreated or treated with the rhMIF (100 ng/ml) for $30 \mathrm{~min}$. The activation of the RhoA and Rac1 GTPases was assessed by G-LISA assays. The data are shown as the mean \pm SD of independent experiments, $\mathrm{n}=3$. (B) After no treatment or exposure to rhMIF for $24 \mathrm{~h}$, the actin filaments of U87 and U251 cells were stained with rhodamine-phalloidin (red), and nuclei were stained with DAPI (blue). Representative magnifications are shown. (C) U87 and U251 cells were subjected to different concentrations of MIF (5, $10,50,100$ and $200 \mathrm{ng} / \mathrm{ml}$ ) for $12 \mathrm{~h}$. The expression levels of p-MYPT1, MYPT1, ROCK1 and GAPDH were determined by western blot analysis. "P<0.05, Student's two-tailed t-test. MIF, macrophage migration inhibitory factor; ROCK, Rho-associated coiled-coil containing kinase; rhMIF, recombinant human MIF; SD, standard deviation.

was enhanced by MIF in glioblastoma cells. G-LISA assays were performed to measure the Rho GTPase activity. The RhoA GTPase activity increased following stimulation with rhMIF, but Racl GTPase was not affected in glioblastoma cells (Fig. 1A). Next, three GBM cell lines were treated with rhMIF, and actin filaments were detected by rhodamine-phalloidin staining. Actin polymerization was strongly enhanced in GBM cells treated with rhMIF as there was increased F-actin stress fibre formation compared to that of the control, indicating that ROCK1 was activated by rhMIF (Fig. 1B). To further confirm the activity of ROCK1, the phosphorylation of MYPT1 was assessed by western blot analysis. MIF significantly increased the phosphorylation of the MYPT1 in a dose-dependent manner (Fig. 1C). In contrast, the expression of ROCK1 was unchanged following rhMIF stimulation, suggesting that ROCK1 was regulated only at the enzyme level. These data demonstrated that MIF activates the canonical RhoA-ROCK1 pathway.

MIF influences autophagy levels in glioma. To explore the role of MIF in autophagic activity in glioblastoma, we performed LC3 conversion and GFP-LC3 puncta formation assays in both
U87 and U251 cell lines. siMIF used to neutralize the level of endogenous MIF was transfected into U87 and U251 cells. The expression of LC3B-II decreased, suggesting that MIF knockdown suppressed autophagy in glioblastoma cells (Fig. 2A). We also examined the localization of GFP-LC3 by fluorescence microscopy in siMIF-transfected U87 and U251 cells stably expressing the GFP-LC3 fusion protein. There was a significant decrease in GFP-LC3 puncta in siMIF-transfected cells compared with that in the negative control cells (Fig. 2B). Both cell lines were stimulated by rhMIF, and autophagy was enhanced, as indicated by the increased expression of LC3B-II and percentage of GFP-LC3 puncta-positive cells (Fig. 2A and B). However, the effect of rhMIF on autophagy of glioblastoma cells seemed not to be quite in a dose-dependent manner.

Finally, to assess the clinical relevance of the above observations, we investigated the expression of MIF and LC3B in glioma specimens. Glioma tissues with strong MIF immunohistochemical signals had high expression of LC3B and vice versa (Fig. 2C). The linear regression analysis indicated the positive correlation of MIF with LC3B. These results demonstrated a positive relationship between MIF and autophagy in glioma. 
A

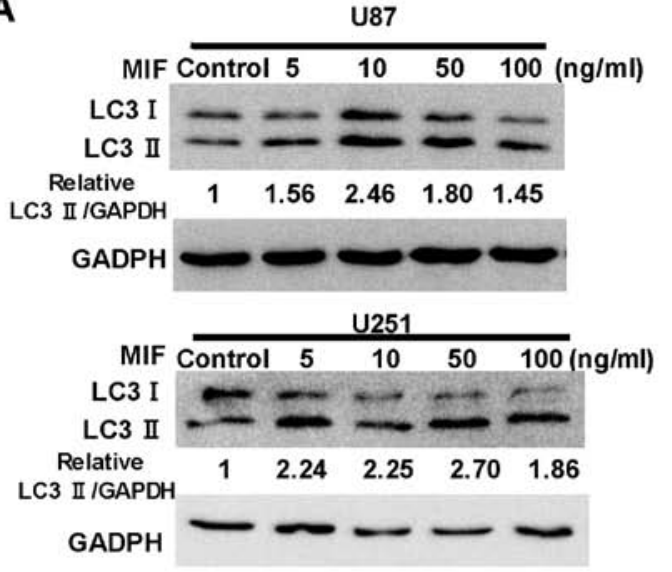

B
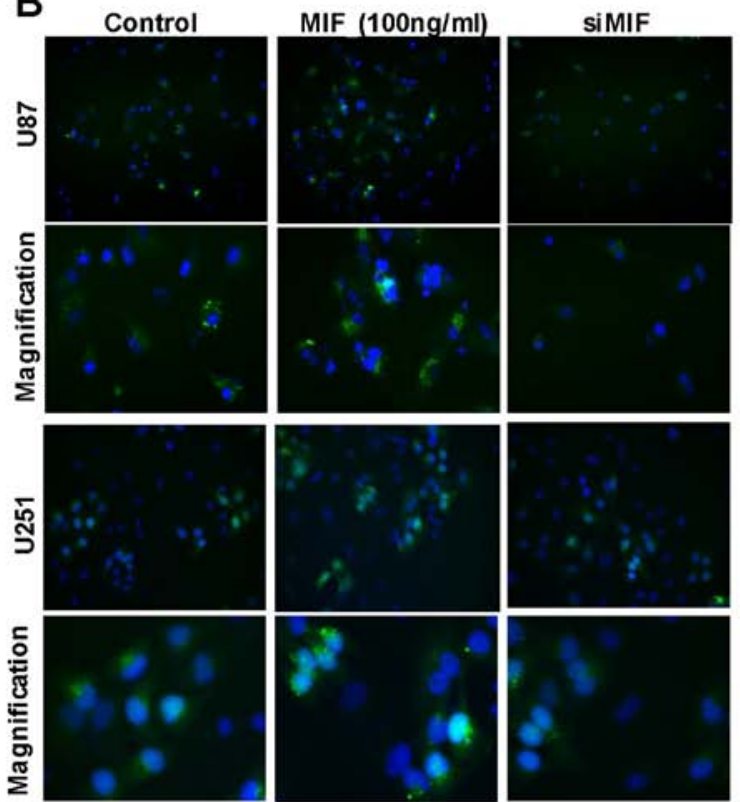

C

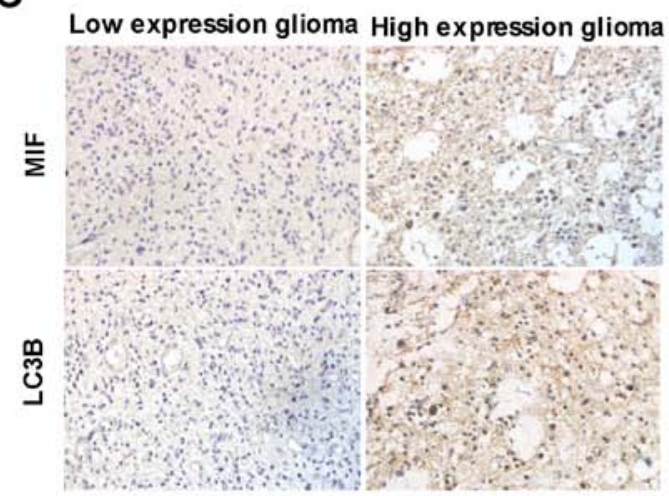

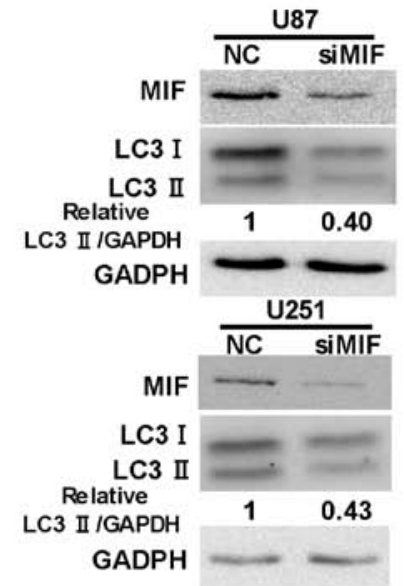
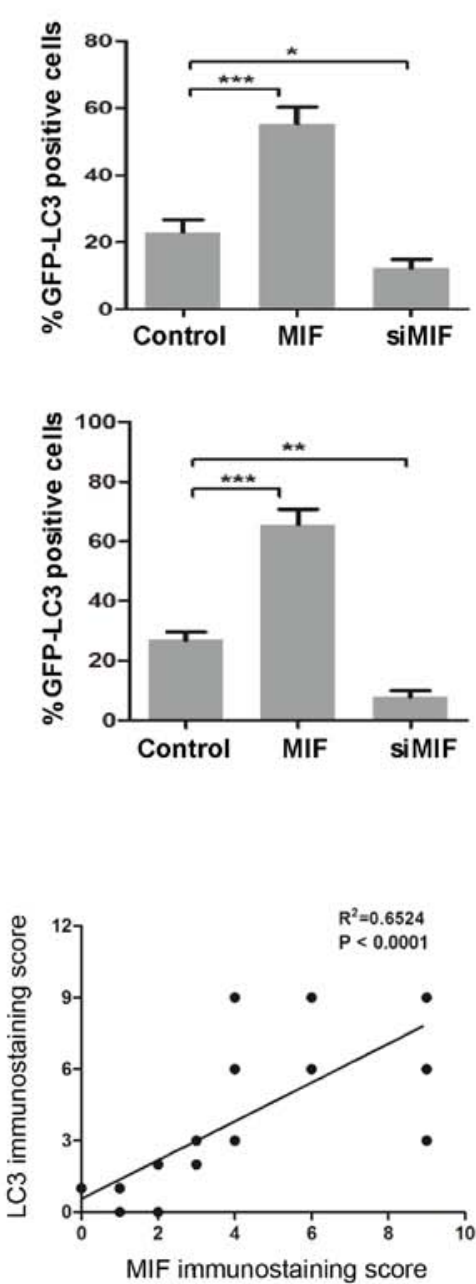

Figure 2. MIF influences the autophagic activity in glioblastoma. (A) U87 and U251 cells were exposed to different concentrations of rhMIF (5, 10, 50 and $100 \mathrm{ng} / \mathrm{ml}$ ) for $24 \mathrm{~h}$. U87 and U251 cells were transfected with siMIF (100 nM) for $24 \mathrm{~h}$. Then, MIF, LC3B and GAPDH levels were determined by western blot analysis. (B) U87 and U251 cells stably expressing GFP-LC3 were stimulated with rhMIF or transfected with siMIF for $24 \mathrm{~h}$ and then fixed. Representative images are shown. Quantitative analysis of the rates of GFP-LC3 puncta-positive cells is shown. The data are shown as the mean \pm SD of independent experiments, $n=3$. (C) Representative images of glioma tissues showed immunostaining of MIF and LC3B (magnification, $x 400$ ). Correlation of MIF expression with LC3B was analyzed in 25 glioma specimens. The linear regression coefficient and statistical significance are shown. ${ }^{*} \mathrm{P}<0.05,{ }^{* *} \mathrm{p}<0.01$, **** $<$ 0.001, one-way ANOVA. MIF, macrophage migration inhibitory factor; rhMIF, recombinant human MIF; SD, standard deviation.

MIF enhances the autophagy, migration and colony formation of glioblastoma cells by activating ROCK1. Although several studies have identified ROCK1 as a regulator of autophagy, whether MIF enhances autophagy via ROCK1 is unknown.
Therefore, we used Y27632, an inhibitor of ROCK1 activity, and siROCK1 to confirm the role of ROCK1 in MIF-induced autophagy. Western blot analyses were performed to detect autophagy. The increased expression of LC3B-II induced 


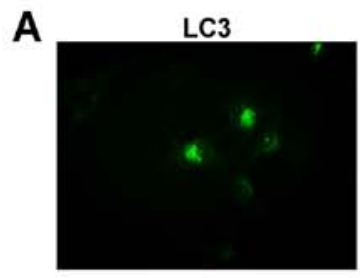

DAPI
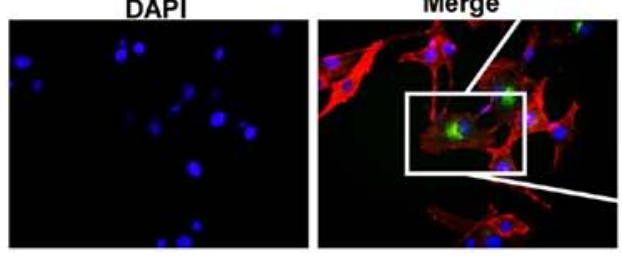

Phalloidin

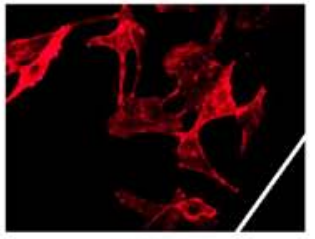

Merge

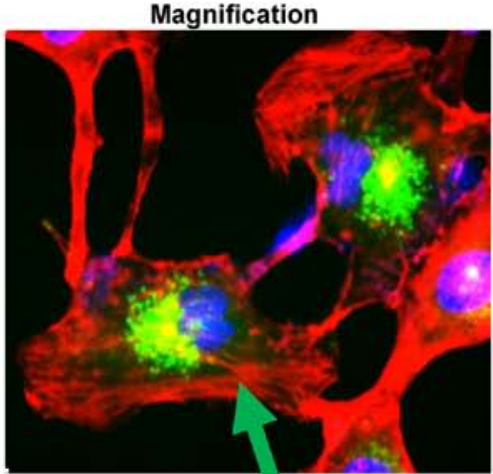

Stress fiber
B

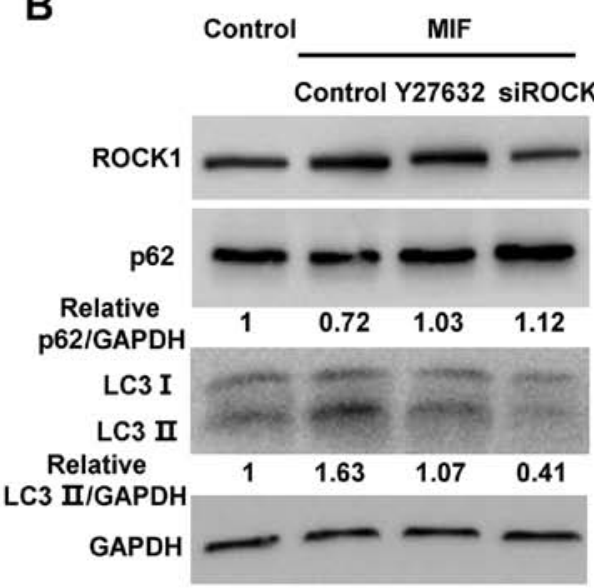

C Control

s.
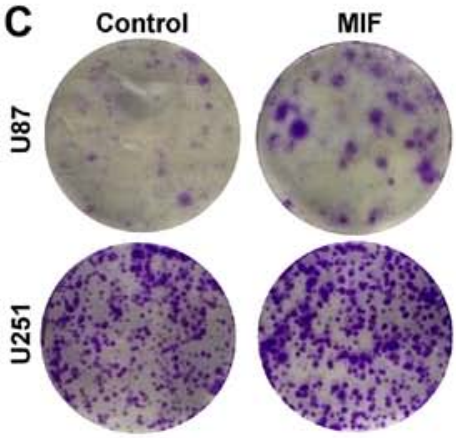

MIF+Y27632

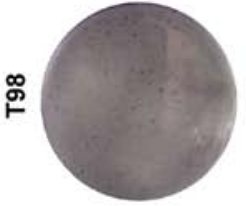

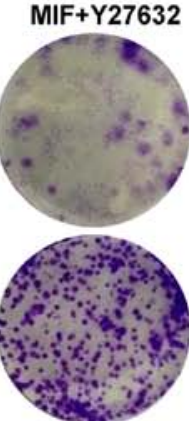
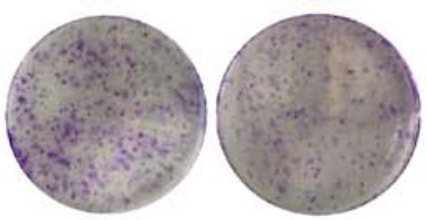

D

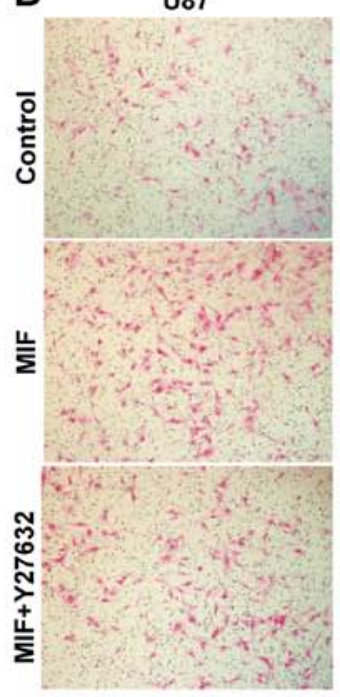

T98

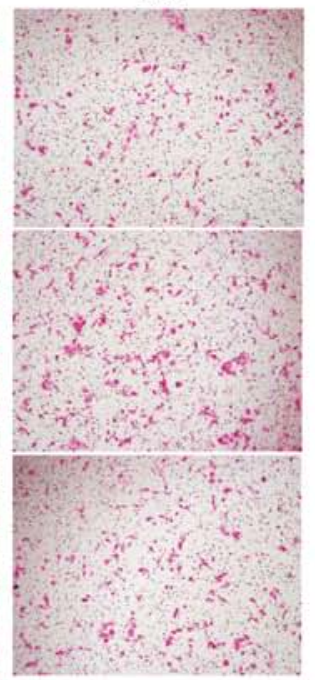

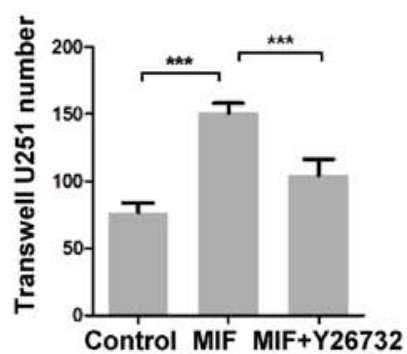

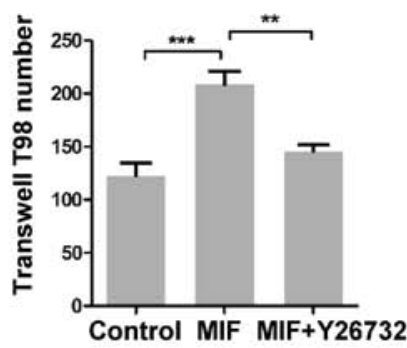

Figure 3. MIF increases autophagy by activating ROCK1. (A) U87 cells stably expressing GFP-LC3 were cultured with MIF for $24 \mathrm{~h}$ and fixed. The actin filaments were stained by rhodamine-phalloidin. The nuclei were stained by DAPI. Representative images are shown. (B) U87 cells were treated with rhMIF, and the activity of ROCK1 was inhibited by Y260072 or siROCK1. LC3B, p62 and GAPDH expressions were detected by western blot analysis. (C) Colony formation assays were performed using U87, U251 and T98 cells stimulated by MIF (10 ng/ml) with or without Y270026 for 4 weeks. Representative images are shown. (D) U87 and T98 cells exposed to MIF $(100 \mathrm{ng} / \mathrm{ml})$ with or without Y270062 were used to perform Transwell assays for $18 \mathrm{~h}$. Representative images are shown. The data are shown as the mean $\pm \mathrm{SD}$ of independent experiments, $\mathrm{n}=3 .{ }^{* *} \mathrm{P}<0.01,{ }^{* * * *} \mathrm{p}<0.001$, one-way ANOVA. MIF, macrophage migration inhibitory factor; ROCK, Rho-associated coiled-coil containing kinase; rhMIF, recombinant human MIF; SD, standard deviation.

by MIF was significantly reversed by Y27632 and ROCK1 knockdown (Fig. 3B). In addition, U87 cells stably expressing
GFP-LC3 were treated with MIF and then fixed to assess the actin filaments by rhodamine-phalloidin staining. The F-actin 


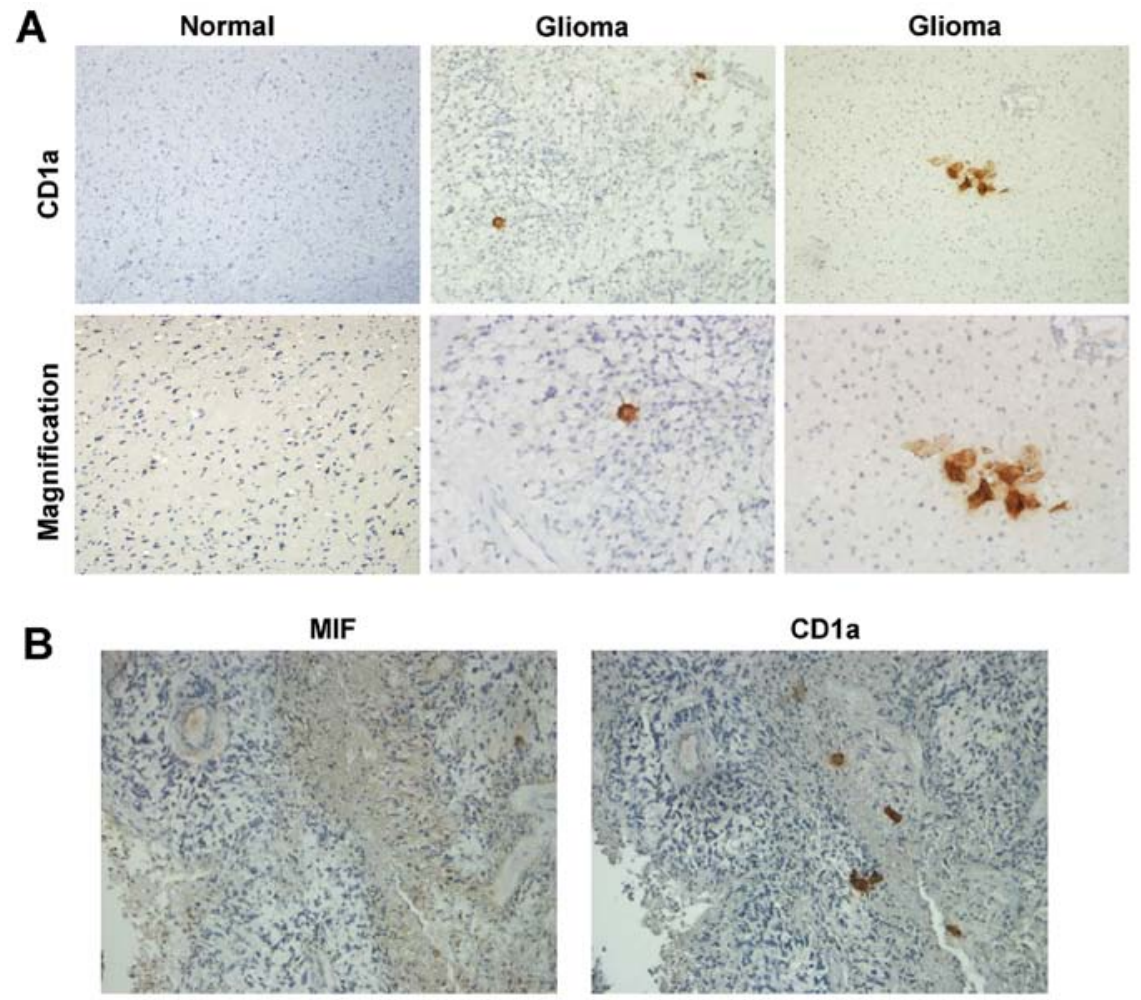

Figure 4. DC infiltrations were detected in glioma tissues. (A) Representative images of glioma and normal brain tissues showed immunostaining of CD1a (magnification, x400). (B) Immunohistochemistry was performed to detect CD1a and MIF in serial sections of GBM (magnification, x200). DC, dendritic cell; MIF, macrophage migration inhibitory factor; GBM, glioblastoma multiforme.

stress fibre formation was localized in GFP-LC3 puncta-positive cells, suggesting that glioblastoma cells with high ROCK1 activity had increased autophagy (Fig. 3A).

Furthermore, we explored whether ROCK1 activity contributes to increased migration and colony formation induced by MIF. The migration of U87, U251 and T98 cells was measured by Transwell assays. rhMIF promoted the migration of all three cell lines, and as expected, Y27632 suppressed the MIF-induced migration (Fig. 3D). Y27632 partly blocked the MIF-induced colony formation (Fig. 3C). These data demonstrated that the activity of ROCK1 played a crucial role in MIF-induced tumourigenesis.

DCs infiltrate glioma tissues. A previous report identified DCs in brain tissues (33). To determine whether DCs infiltrated glioma tissues, we used CD1a, a marker of DCs, to detect DCs in glioma specimens by immunohistochemistry (34). Few CD1a-positive cells were found in glioma specimens, while in normal brain tissues, there were no CD1a-positive cells (Fig. 4A). This suggested that DCs could infiltrate gliomas through blood-brain barrier (BBB) disruption or an unknown mechanism. Moreover, in serial sections of glioma tissue, CD1a-positive cells were localized in regions with high levels of MIF (Fig. 4B). These data indicated that MIF may suppress the migration of DCs to impede initiation of immune responses.

MIF inhibits the migration of both iDCs and mDCs. To study the effects of MIF on DCs, we generated iDCs and mDCs from PBMCs of healthy individuals (Fig. 5A). The cellular morphology of the iDCs and mDCs was observed (Fig. 5B). The effect of MIF on migration of DCs was assessed using Transwell assays. The migration of iDCs and mDCs towards CCL21 was significantly decreased in MIF-treated DCs compared to that of the controls (Fig. 5C). Furthermore, to verify whether MIF regulates actin rearrangement in DCs, rhodamine-phalloidin staining was performed. There were strong phalloidin-stained puncta in MIF-treated DCs, suggesting a deficiency of the intact F-actin ring (Fig. 5D).

MIF counteracts iDC maturation and mDC function. To further characterize the immunological impact of MIF on DCs, we studied its effects on DC maturation and the expression of co-stimulatory molecules. iDCs were cultured with MIF or supernatants from siMIF-transfected or negative control U87 cells for 3 days in the presence of LPS, which promoted maturation (Fig. 6A). Subsequently, the expression of the maturation markers CD83, CD80 and CD86 was quantified by qPCR. In the presence of MIF, the two co-stimulatory molecule markers, but not CD83, were significantly decreased compared to that of the no MIF treatment, indicating that MIF had an inhibitory role in the maturation of iDCs (Fig. 6C). Similarly, cultures of iDCs with supernatant from siMIF-transfected U87 cells showed slightly increased expression of CD80 (Fig. 6C), while increased expression of CD83 and CD86 was not detected (data not shown). Moreover, we cultured the mDCs in the presence or absence of MIF for $24 \mathrm{~h}$, and the expression of the markers was also downregulated (Fig. 6B). These data demonstrated that MIF plays an inhibitory role in DC maturation and function. 


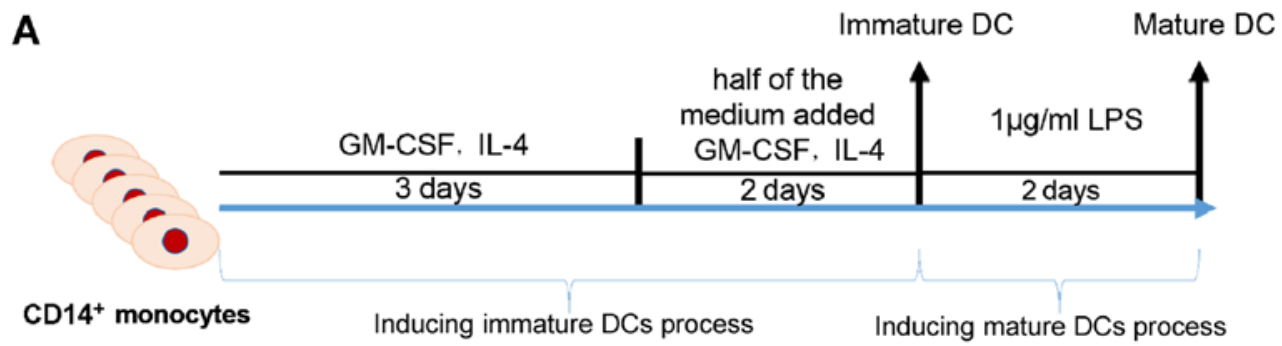

B

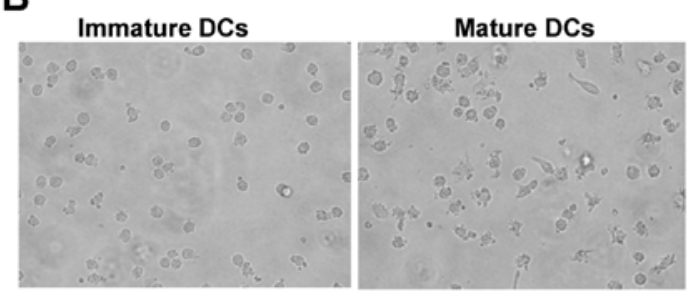

D

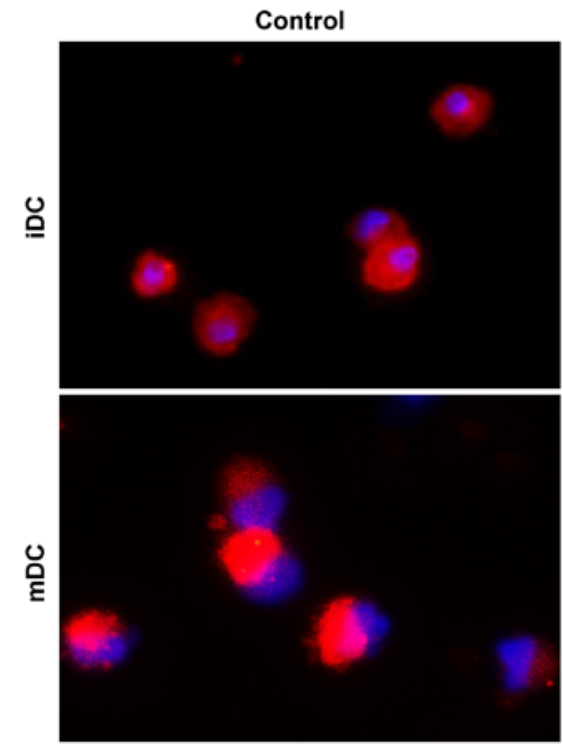

C
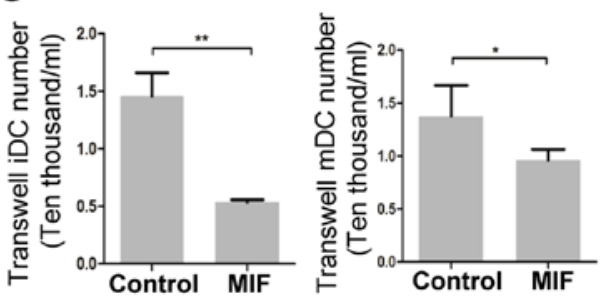

MIF
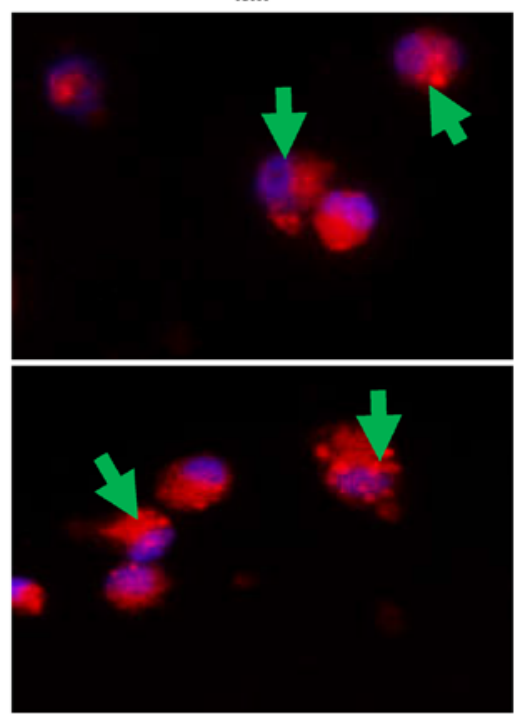

Figure 5. MIF suppresses the migration of DCs. (A) A schematic diagram of the induction of iDCs and mDCs is shown. (B) Images of iDCs and mDCs are exhibited (magnification, $x 400$ ). (C) iDCs and mDCs were treated with rhMIF, and Transwell assays were performed. The data are shown as the mean \pm SD of independent experiments, $\mathrm{n}=3$. (D) iDCs and $\mathrm{mDC}$ sere stained by rhodamine-phalloidin. Representative images are shown. ${ }^{*} \mathrm{P}<0.05,{ }^{* *} \mathrm{p}<0.01, \mathrm{Student}$ 's two-tailed t-test. MIF, macrophage migration inhibitory factor; DCs, dendritic cells; iDCs, immature DCs; mDCs, mature DCs; rhMIF, recombinant human MIF; SD, standard deviation.

\section{Discussion}

MIF is a pleiotropic cytokine that has important pro-inflammatory and pro-tumourigenesis roles. Intriguingly, increasing data have attributed the immune evasion of glioblastoma to MIF. The involvement of MIF in both tumourigenesis and tumour immune escape indicates that it may be a promising target for anti-glioblastoma treatment. In this study, we found that MIF augmented autophagy of glioblastoma cells by activating the RhoA-ROCK1 pathway. In addition, we identified the inhibitory immunological effect of MIF on DCs.

Overexpression of MIF is associated with malignancy, recurrence and poor prognosis of patients with gliomas (35). Extensive studies have shown that MIF contributes to malignant progression of glioma via various processes, including angiogenesis, migration, invasion and contact inhibition. Most recently, MIF has been shown to facilitate brain tumourigenesis (36) and maintain the tumourigenic capacity of brain tumour-initiating cells (37), which supports its pro-tumourigenic role in glioma. Autophagy as a process of cellular self-digestion has been considered a pro-survival response to glioma treatment $(38,39)$. MIF was associated with autophagy. However, to date, whether MIF is implicated in autophagy and its underlying mechanism has not yet been determined in glioma.

There are conflicting reports on whether MIF increases or decreases autophagy in other tumours. In breast cancer, $\mathrm{Wu}$ et al demonstrated that MIF is a strong suppressor of autophagy, leading to resistance to chemotherapy-induced autophagic cell death (40). In contrast, several studies showed that MIF induced autophagy via reactive oxygen species generation and contributed to anti-concanavalin A-induced apoptosis by upregulating autophagy $(41,42)$. Moreover, MIF knockout was associated with loss in cardiac autophagy during ageing (43). In our study, we found that exogenous 
A MIF, supernatant of U87, hypoxia

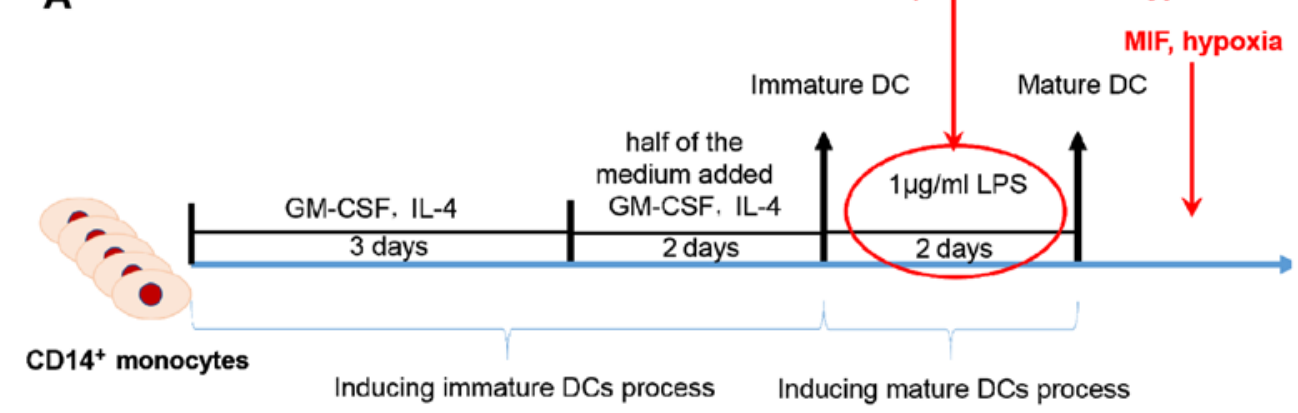

B

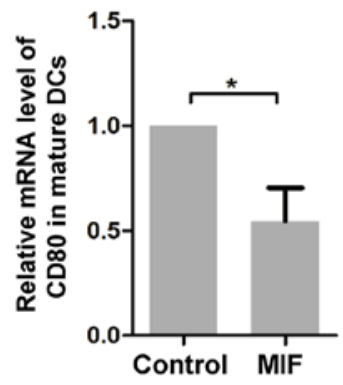

C

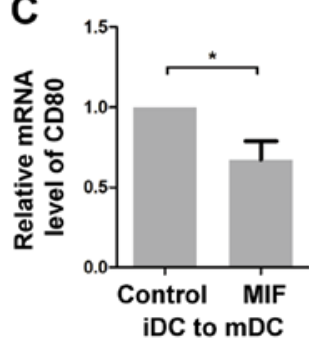

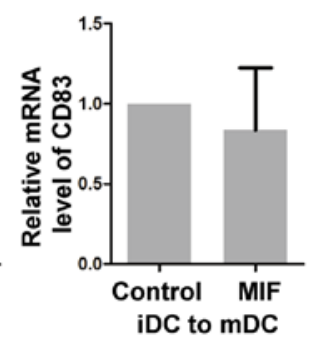
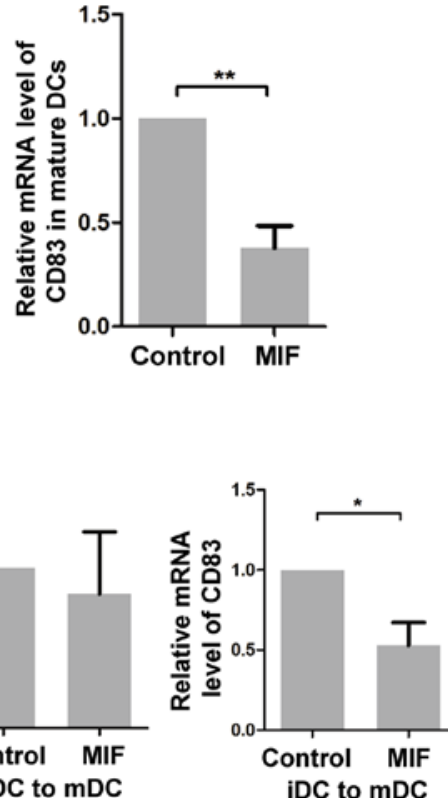
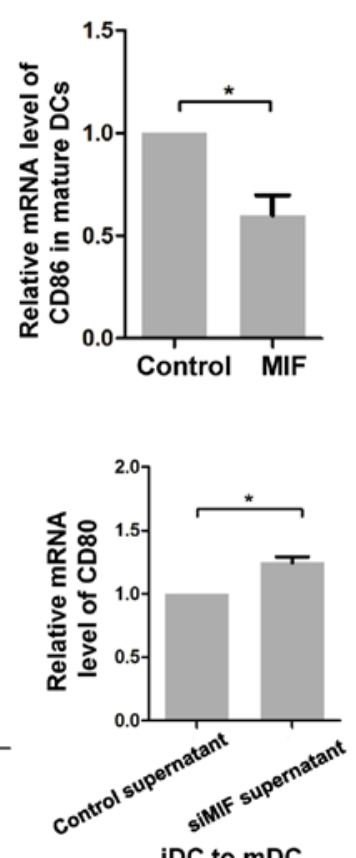

Figure 6. MIF suppresses the maturation and function of DCs. (A) A schematic diagram of the intervention during or after the induction of mDCs from iDCs is shown. (B) mDCs were treated with rhMIF for $24 \mathrm{~h}$. Cells were collected for qPCR analysis to quantify the expression of CD80, CD83 and CD86. The data are shown as the mean $\pm \mathrm{SD}$ of independent experiments, $n=3$. (C) rhMIF was added as indicated during the maturation of iDCs, and the DCs were then collected to measure the expression of CD80, CD83 and CD86. The data are shown as the mean \pm SD of independent experiments, $n=3$. ${ }^{*} \mathrm{P}<0.05,{ }^{* * *} \mathrm{p}<0.01$, Student's two-tailed t-test. MIF, macrophage migration inhibitory factor; DCs, dendritic cells; mDCs, mature DCs; iDCs, immature DCs; rhMIF, recombinant human MIF; SD, standard deviation.

MIF promoted autophagy in glioblastoma cells. Since the identification of MIF, it has been reported to be involved in pleiotropic cytokine signalling via multiple receptors, such as CD74, CD44 and CXC chemokine receptors (44-46). The opposing effects of autophagy in different cells may be attributed to various receptor patterns. Interestingly, Rho GTPases have been reported to be involved in MIF-induced tumour invasion $(47,48)$. Most importantly, ROCK1, the downstream effector of the RhoA, was identified as a critical regulator of autophagy during metabolic stress (49). Our study confirmed that MIF increased the RhoA-ROCK1 activation but not the expression level of ROCK1, as determined by the G-LISA assay and the phosphorylation of MYPT1. Moreover, ROCK1 has been shown to be involved in the migration and proliferation of glioblastoma $(50,51)$. These results led us to hypothesize that MIF enhances autophagy, and even malignant progression, by activating ROCK1 in glioma. Knockdown of ROCK1 or inhibition of ROCK1 reversed the autophagy, migration and colony formation induced by MIF. Interestingly, although
U251 showed higher ability of colony formation in the control group than the other two cell lines, U251 was also significantly influenced by MIF stimulation and the ROCK1 activity. These results demonstrate the important role of ROCK1 in regulating the MIF-induced malignant progression of glioma.

Although therapeutic strategies have improved in the past decades, the average overall survival for patients with glioblastoma is still very poor (52). Immune therapy has attracted increased attention and has emerged as a promising adjuvant treatment for glioblastoma. There is clear evidence for the immune escape role of MIF in gliomas $(53,54)$. In glioma, MIF suppressed the antitumoural effects of NK cells and downregulated the activating immune receptor (NKG2D) on NK cells and $\mathrm{CD} 8^{+} \mathrm{T}$ cells. In addition, a recent study showed that MIF suppressed the immune response by supporting immune-suppressive myeloid-derived suppressor cells in the GBM tumour microenvironment. However, the association between DCs, initiators of the immune response, and MIF remains unclear. First, we found infiltration of DCs in gliomas. 
Intriguingly, CDla-positive cells were localized in regions with high expression of MIF, suggesting an inhibitory effect of MIF on migration of DCs. To address this hypothesis, we sorted the $\mathrm{CD} 14^{+}$monocytes from PBMCs and used them to generate iDCs and mDCs. Transwell assays confirmed that MIF had a strong inhibitory effect on the migration of both iDCs and mDCs. Additionally, the intact F-actin ring appeared to be dysfunctional with MIF treatment. Because DCs migrate through pathological tissues before reaching their final destination in the lymph nodes, the inhibitory effect of MIF can delay or prevent DCs from returning to the lymph nodes to stimulate the immune response. Considering that DC maturation is required for the initiation of an immune response, the effect of MIF on maturation and function of DCs was measured by assessing the maturation marker, CD83, and co-stimulatory molecules (CD80 and CD86) (55). Accordingly, incubation of mDCs with MIF substantially decreased the expression of CD83, CD80 and CD86, reflecting a significant immunosuppressive effect of MIF on mDCs. In addition, MIF apparently impaired the LPS-induced maturation of DCs, confirmed by the decreased expression of co-stimulatory molecules, but not CD83. To assess the glioma microenvironment, we used the supernatant of U87 cells, which has been reported to have higher MIF RNA levels than U251 cells, for further experiments. Consistent with previous observations, incubation of iDCs cultured with LPS and supernatant from knockdown MIF U87 cells slightly augmented the expression of CD80 compared to that of the control supernatant. The supernatant contains much lower concentrations of MIF than the exogenous MIF, indicating that CD80 was more sensitive to the effect of MIF.

In summary, we showed that MIF is a critical mediator of autophagy in glioblastoma. We identified ROCK1 as a potent downstream effector of MIF that further regulates autophagy. Moreover, ROCK1 may also be a core regulator that promotes MIF-induced malignant progression of glioblastoma. In addition, we showed that MIF plays an important role in the escape of DC surveillance. It suppressed the maturation and function of DCs, which was reflected by the downregulation of the maturation marker CD83 and the co-stimulatory molecules CD80 and CD86. However, the detailed mechanism underlying the inhibitory effect of MIF on DCs was not elucidated in this study, and further investigations should be performed to define this mechanism. Finally, we showed that MIF directly or indirectly contributes to an immune microenvironment that favours glioblastoma progression.

\section{Acknowledgements}

We thank Professor Xun Qu for helpful comments and advice on this study. This study was supported by grants from the National Natural Science Foundation of China (nos. 81101594, $81372719,81172403,81300510,81402077,81571284$ and 91542115) and Taishan Scholars of Shandong Province of China (no. ts201511093).

\section{References}

1. Meyer MA: Malignant gliomas in adults. N Engl J Med 359: 1850; author reply 1850, 2008.
2. Ohgaki $\mathrm{H}$ and Kleihues P: Population-based studies on incidence, survival rates, and genetic alterations in astrocytic and oligodendroglial gliomas. J Neuropathol Exp Neurol 64: 479-489, 2005.

3. Bloom BR and Bennett B: Mechanism of a reaction in vitro associated with delayed-type hypersensitivity. Science 153: 80-82, 1966.

4. Calandra $\mathrm{T}$ and Roger T: Macrophage migration inhibitory factor: A regulator of innate immunity. Nat Rev Immunol 3: 791-800, 2003.

5. Apte RS, Sinha D, Mayhew E, Wistow GJ and Niederkorn JY: Cutting edge: Role of macrophage migration inhibitory factor in inhibiting NK cell activity and preserving immune privilege. J Immunol 160: 5693-5696, 1998.

6. Daun JM and Cannon JG: Macrophage migration inhibitory factor antagonizes hydrocortisone-induced increases in cytosolic IkappaBalpha. Am J Physiol Regul Integr Comp Physiol 279: R1043-R1049, 2000.

7. Bucala R and Donnelly SC: Macrophage migration inhibitory factor: A probable link between inflammation and cancer. Immunity 26: 281-285, 2007.

8. Roger T, Chanson AL, Knaup-Reymond M and Calandra T: Macrophage migration inhibitory factor promotes innate immune responses by suppressing glucocorticoid-induced expression of mitogen-activated protein kinase phosphatase-1. Eur J Immunol 35: 3405-3413, 2005.

9. Meyer-Siegler KL, Iczkowski KA, Leng L, Bucala R and Vera PL: Inhibition of macrophage migration inhibitory factor or its receptor (CD74) attenuates growth and invasion of DU-145 prostate cancer cells. J Immunol 177: 8730-8739, 2006.

10. Bacher M, Eickmann M, Schrader J, Gemsa D and Heiske A: Human cytomegalovirus-mediated induction of MIF in fibroblasts. Virology 299: 32-37, 2002.

11. Mitchell RA, Metz CN, Peng T and Bucala R: Sustained mitogen-activated protein kinase (MAPK) and cytoplasmic phospholipase $\mathrm{A} 2$ activation by macrophage migration inhibitory factor (MIF). Regulatory role in cell proliferation and glucocorticoid action. J Biol Chem 274: 18100-18106, 1999.

12. Bacher M, Schrader J, Thompson N, Kuschela K, Gemsa D, Waeber G and Schlegel J: Up-regulation of macrophage migration inhibitory factor gene and protein expression in glial tumor cells during hypoxic and hypoglycemic stress indicates a critical role for angiogenesis in glioblastoma multiforme. Am J Pathol 162: 11-17, 2003.

13. Lue H, Thiele M, Franz J, Dahl E, Speckgens S, Leng L, Fingerle-Rowson G, Bucala R, Lüscher B and Bernhagen J: Macrophage migration inhibitory factor (MIF) promotes cell survival by activation of the Akt pathway and role for CSN5/JAB1 in the control of autocrine MIF activity. Oncogene 26: 5046-5059, 2007.

14. Rabinowitz JD and White E: Autophagy and metabolism. Science 330: 1344-1348, 2010.

15. Mizushima $\mathrm{N}$ and Komatsu M: Autophagy: Renovation of cells and tissues. Cell 147: 728-741, 2011.

16. Shintani T and Klionsky DJ: Autophagy in health and disease: A double-edged sword. Science 306: 990-995, 2004.

17. Aita VM, Liang XH, Murty VV, Pincus DL, Yu W, Cayanis E, Kalachikov S, Gilliam TC and Levine B: Cloning and genomic organization of beclin 1, a candidate tumor suppressor gene on chromosome 17q21. Genomics 59: 59-65, 1999.

18. Choi AM, Ryter SW and Levine B: Autophagy in human health and disease. N Engl J Med 368: 1845-1846, 2013.

19. Liang XH, Jackson S, Seaman M, Brown K, Kempkes B, Hibshoosh $\mathrm{H}$ and Levine B: Induction of autophagy and inhibition of tumorigenesis by beclin 1. Nature 402: 672-676, 1999 .

20. Guo JY, Xia B and White E: Autophagy-mediated tumor promotion. Cell 155: 1216-1219, 2013.

21. Hall A: The cytoskeleton and cancer. Cancer Metastasis Rev 28: 5-14, 2009.

22. Matsui T, Amano M, Yamamoto T, Chihara K, Nakafuku M, Ito M, Nakano T, Okawa K, Iwamatsu A and Kaibuchi K: Rho-associated kinase, a novel serine/threonine kinase, as a putative target for small GTP binding protein Rho. EMBO J 15: 2208-2216, 1996.

23. Leung T, Manser E, Tan L and Lim L: A novel serine/threonine kinase binding the Ras-related RhoA GTPase which translocates the kinase to peripheral membranes. J Biol Chem 270: 29051-29054, 1995.

24. Amano M, Chihara K, Kimura K, Fukata Y, Nakamura N, Matsuura $Y$ and Kaibuchi K: Formation of actin stress fibers and focal adhesions enhanced by Rho-kinase. Science 275: 1308-1311, 1997. 
25. Riento K and Ridley AJ: Rocks: Multifunctional kinases in cell behaviour. Nat Rev Mol Cell Biol 4: 446-456, 2003.

26. Amano M, Nakayama M and Kaibuchi K: Rho-kinase/ROCK: A key regulator of the cytoskeleton and cell polarity. Cytoskeleton Hoboken 67: 545-554, 2010.

27. Banchereau J and Steinman RM: Dendritic cells and the control of immunity. Nature 392: 245-252, 1998.

28. Lai A, Tran A, Nghiemphu PL, Pope WB, Solis OE, Selch M, Filka E, Yong WH, Mischel PS, Liau LM, et al: Phase II study of bevacizumab plus temozolomide during and after radiation therapy for patients with newly diagnosed glioblastoma multiforme. J Clin Oncol 29: 142-148, 2011.

29. Ghulam Muhammad AK, Candolfi M, King GD, Yagiz K, Foulad D, Mineharu Y, Kroeger KM, Treuer KA, Nichols WS Sanderson NS, et al: Antiglioma immunological memory in response to conditional cytotoxic/immune-stimulatory gene therapy: Humoral and cellular immunity lead to tumor regression. Clin Cancer Res 15: 6113-6127, 2009.

30. Perng $\mathrm{P}$ and Lim M: Immunosuppressive mechanisms of malignant gliomas: Parallels at non-CNS sites. Front Oncol 5 : $153,2015$.

31. Van Gool S, Maes W, Ardon H, Verschuere T, Van Cauter S and De Vleeschouwer S: Dendritic cell therapy of high-grade gliomas. Brain Pathol 19: 694-712, 2009.

32. Shao Q, Ning H, Lv J, Liu Y, Zhao X, Ren G, Feng A, Xie Q, Sun J, Song B, et al: Regulation of Th1/Th2 polarization by tissue inhibitor of metalloproteinase-3 via modulating dendritic cells. Blood 119: 4636-4644, 2012.

33. Laperchia C, Allegra Mascaro AL, Sacconi L, Andrioli A, Mattè A, De Franceschi L, Grassi-Zucconi G, Bentivoglio M, Buffelli M and Pavone FS: Two-photon microscopy imaging of thylGFP-M transgenic mice: A novel animal model to investigate brain dendritic cell subsets in vivo. PLoS One 8: e56144, 2013.

34. Coventry BJ, Austyn JM, Chryssidis S, Hankins D and Harris A: Identification and isolation of CD1a positive putative tumour infiltrating dendritic cells in human breast cancer. Adv Exp Med Biol 417: 571-577, 1997.

35. Wang XB, Tian XY, Li Y, Li B and Li Z: Elevated expression of macrophage migration inhibitory factor correlates with tumor recurrence and poor prognosis of patients with gliomas. J Neurooncol 106: 43-51, 2012.

36. Ghoochani A, Schwarz MA, Yakubov E, Engelhorn T, Doerfler A, Buchfelder M, Bucala R, Savaskan NE and Eyüpoglu IY: MIF-CD74 signaling impedes microglial M1 polarization and facilitates brain tumorigenesis. Oncogene: May 9, 2016 (Epub ahead of print)

37. Fukaya R, Ohta S, Yaguchi T, Matsuzaki Y, Sugihara E, Okano H, Saya H, Kawakami Y, Kawase T, Yoshida K, et al: MIF maintains the tumorigenic capacity of brain tumor-initiating cells by directly inhibiting p53. Cancer Res 76: 2813-2823, 2016

38. Xue H, Yuan G, Guo X, Liu Q, Zhang J, Gao X, Guo X, Xu S, $\mathrm{Li}$ T, Shao Q, et al: A novel tumor-promoting mechanism of IL6 and the therapeutic efficacy of tocilizumab: Hypoxia-induced IL6 is a potent autophagy initiator in glioblastoma via the p-STAT3-MIR155-3p-CREBRF pathway. Autophagy 12 : 1129-1152, 2016.

39. Liu R, Li J,Zhang T,Zou L, Chen Y, Wang K, Lei Y, Yuan K, Li Y, Lan J, et al: Itraconazole suppresses the growth of glioblastoma through induction of autophagy: Involvement of abnormal cholesterol trafficking. Autophagy 10: 1241-1255, 2014.

40. Wu MY, Fu J, Xu J, O'Malley BW and Wu RC: Steroid receptor coactivator 3 regulates autophagy in breast cancer cells through macrophage migration inhibitory factor. Cell Res 22: 1003-1021, 2012.
41. Lai YC, Chuang YC, Chang CP and Yeh TM: Macrophage migration inhibitory factor has a permissive role in concanavalin A-induced cell death of human hepatoma cells through autophagy. Cell Death Dis 6: e2008, 2015.

42. Chuang YC, Su WH, Lei HY, Lin YS, Liu HS, Chang CP and Yeh TM: Macrophage migration inhibitory factor induces autophagy via reactive oxygen species generation. PLoS One 7: e37613, 2012.

43. Xu X, Hua Y, Nair S, Bucala R and Ren J: Macrophage migration inhibitory factor deletion exacerbates pressure overload-induced cardiac hypertrophy through mitigating autophagy. Hypertension 63: 490-499, 2014.

44. Leng L, Metz CN, Fang Y, Xu J, Donnelly S, Baugh J, Delohery T, Chen Y, Mitchell RA and Bucala R: MIF signal transduction initiated by binding to CD74. J Exp Med 197: 1467-1476, 2003.

45. Shi X, Leng L, Wang T, Wang W, Du X, Li J, McDonald C, Chen Z, Murphy JW, Lolis E, et al: CD44 is the signaling component of the macrophage migration inhibitory factor-CD74 receptor complex. Immunity 25: 595-606, 2006.

46. Bernhagen J, Krohn R, Lue H, Gregory JL, Zernecke A, Koenen RR, Dewor M, Georgiev I, Schober A, Leng L, et al: MIF is a noncognate ligand of CXC chemokine receptors in inflammatory and atherogenic cell recruitment. Nat Med 13: 587-596, 2007.

47. Rendon BE, Roger T, Teneng I, Zhao M, Al-Abed Y, Calandra T and Mitchell RA: Regulation of human lung adenocarcinoma cell migration and invasion by macrophage migration inhibitory factor. J Biol Chem 282: 29910-29918, 2007.

48. Sun B, Nishihira J, Yoshiki T, Kondo M, Sato Y, Sasaki F and Todo S: Macrophage migration inhibitory factor promotes tumor invasion and metastasis via the Rho-dependent pathway. Clin Cancer Res 11: 1050-1058, 2005.

49. Gurkar AU, Chu K, Raj L, Bouley R, Lee SH, Kim YB, Dunn SE, Mandinova A and Lee SW: Identification of ROCK1 kinase as a critical regulator of Beclin1-mediated autophagy during metabolic stress. Nat Commun 4: 2189, 2013.

50. Zhang P, Lu Y, Liu XY and Zhou YH: Knockdown of Rho-associated protein kinase 1 suppresses proliferation and invasion of glioma cells. Tumour Biol 36: 421-428, 2015.

51. Huang D, Qiu S, Ge R, He L, Li M, Li Y and Peng Y: miR-340 suppresses glioblastoma multiforme. Oncotarget 6: 9257-9270, 2015.

52. Omuro A and DeAngelis LM: Glioblastoma and other malignant gliomas: A clinical review. JAMA 310: 1842-1850, 2013.

53. Mittelbronn M, Platten M, Zeiner P, Dombrowski Y, Frank B Zachskorn C, Harter PN, Weller $M$ and Wischhusen $\mathrm{J}$ : Macrophage migration inhibitory factor (MIF) expression in human malignant gliomas contributes to immune escape and tumour progression. Acta Neuropathol 122: 353-365, 2011.

54. Otvos B, Silver DJ, Mulkearns-Hubert EE, Alvarado AG, Turaga SM, Sorensen MD, Rayman P, Flavahan WA, Hale JS, Stoltz K, et al: Cancer stem cell-secreted macrophage migration inhibitory factor stimulates myeloid derived suppressor cell function and facilitates glioblastoma immune evasion. Stem Cells 34: 2026-2039, 2016.

55. Etminan N, Peters C, Lakbir D, Bünemann E, Börger V, Sabel MC, Hänggi D, Steiger HJ, Stummer W and Sorg RV: Heat-shock protein 70 -dependent dendritic cell activation by 5 -aminolevulinic acid-mediated photodynamic treatment of human glioblastoma spheroids in vitro. Br J Cancer 105: 961-969, 2011. 